\title{
Tactile Tracking of Arteries in Robotic Surgery
}

\author{
Ryan A. Beasley and Robert D. Howe \\ Division of Engineering and Applied Sciences \\ Harvard University, Cambridge, Massachusetts 02138 \\ email: rbeasley@deas.harvard.edu,howe@deas.harvard.edu
}

\begin{abstract}
Locating arteries hidden beneath superficial tissue can be a difficult task in minimally invasive surgery. This paper reports the development of a system that finds the paths of arteries using tactile sensing. The surgeon begins by using the surgical robot to place the tactile sensor instrument on a known artery location. Signal processing algorithms locate the artery from its pulsatile pressure variation. An adaptive extrapolation algorithm then generates predicted locations for the artery based on previous measurements. After moving to the predicted location, if the artery is not located then a backtracking mechanism moves the sensor towards previously detected locations. Tests with model arteries show good tracking ability for circular arcs with curvatures as small as $80 \mathrm{~mm}$, although problems with compliance in the system result in occasional loss of the artery path. Preliminary tests demonstrate the ability to transcutaneously track the radial artery in the human wrist.
\end{abstract}

\section{Introduction}

Minimally-invasive surgical techniques make it difficult to locate arteries hidden beneath overlying tissue. This is particularly true in robot-assisted coronary artery bypass grafting $(\mathrm{CABG})$, where the robot is used to mobilize the internal mammary artery (IMA) from the interior chest wall adjacent to the sternum, so that one end may be attached to a coronary artery to provide a new blood supply for the heart (Stephenson et al. 1998). Visual detection of the IMA location is difficult or impossible because the artery is largely hidden beneath the superficial tissue. In the conventional surgical approach where the chest is opened to provide access, the artery may be readily located through palpation, i.e., using the fingertips to detect the pulsatile pressure variations. Unfortunately, fingertip palpation is not possible in minimally invasive procedures, where surgeons work through incisions under $1 \mathrm{~cm}$ in diameter with long-handled instruments or surgical robots. Artery localization must then rely on careful dissection to visually expose the artery, a painstaking process that at present consumes an unacceptably large fraction of the procedure duration.

We are investigating a number of solutions to the problem of locating hidden arteries. We have developed a technique that uses pre-operative CT scans with injected contrast agent to establish the location of the IMA in the 3D CT data set. This location is then registered to the patient's anatomy during the procedure using fiducials or anatomical landmarks, and used to guide instruments to the appropriate dissection path adjacent to the artery (Park, Howe, and Torchiana 2001). While animal trials are promising, this approach is complex: it requires acquisition and processing of a preoperative CT scan with contrast, and accurate registration requires minimal movement between the artery and the adjacent anatomy in the interval between CT scanning and the operation.

To advance beyond this cumbersome approach, we are working to use intraoperative imaging to determine the artery's location. There are two candidate imaging technologies for this application: ultrasound and tactile sensing. Ultrasound is a mature technology with strong contrast for blood flow using Doppler imaging (Schellenberg, Marshall, and Salgo 2001); it is also relatively expensive, and its utility in $\mathrm{CABG}$ procedures is likely limited to IMA localization. Tactile sensing is not as widely established, although it has seen growing applications in surgery (Matsumoto et al. 1997; Peine 1998; Harris et al. 1999). The relative simplicity of implementation, however, and analogy to human palpation recommends tactile sensing for this application. Furthermore, tactile information would be useful throughout a surgical procedure for tasks like determining tissue mechanical properties and perception of grasping and retraction forces, and a number of groups are working to incorporate tactile sensors into a variety of instruments for minimally invasive surgery (Howe et al. 1995; Fischer and Trapp 1996).

In this paper, we present the results of our initial design and laboratory validation of a system for locating arteries in soft tissue. We begin by describing the tactile sensor and signal processing algorithm that detects and localizes the artery's pressure variation on the sensor surface. Next, we present the control algorithm that establishes the artery location and then moves along the tissue surface to follow the artery's path. This system is then tested in a series of experiments that measure its ability to track the artery through in vitro model tests and in vivo trials on the radial artery in the human arm.

\section{System Design and Experimental Methods}

Our system for robotic artery localization consists of three principal components: the tactile sensor and its interface electronics, the signal processing algorithm, and the artery tracking controller for the robot. 


\section{Sensor and interface electronics}

In artery tracking applications like IMA harvest, the tactile sensor slides along a relatively smooth tissue plane. In this case a single line of pressure sensing elements is sufficient, if the artery path is perpendicular to the line of sensor elements. The tactile sensor used in these experiments is cylindrical, with a line of 10 pressure sensing elements on $2 \mathrm{~mm}$ centers along the bottom edge (Figure 1). The capacitive sensor is modeled on a design by Fearing (1990) and consists of two layers of copper strips separated by an elastic dielectric. Applying pressure to the sensor's upper surface forces the copper strips closer together, increasing the capacitance. The change in the measured capacitance of each element is closely proportional to the local pressure above it. The sensor array is mounted on an aluminum cylinder $9 \mathrm{~mm}$ in diameter and $34 \mathrm{~mm}$ in length, with the first sensor located $1 \mathrm{~mm}$ from the distal end of the cylinder. The array was covered with $2 \mathrm{~mm}$ of elastomer (GE RTV 110 Silicone Rubber Adhesive Sealant) to protect it from mechanical damage. The standard deviation of the noise of each element is $0.012 \mathrm{~N}$. For further details of sensor design and performance see (Pawluk et al. 1998) and (Peine 1998).

\section{Artery Detection and Tracking Algorithms}

There are two signal processing tasks in artery tracking. First, the software must differentiate between the artery's pulsatile pressure variation and the background pressure pattern due to other tissue. Second, the position of the artery on the sensor must be determined and reported to the robot controller. The tracking process also has two distinct phases: the initial problem is locating one spot on the artery to start the tracking process, succeeded by the problem of determining motions to follow the artery's course.

Because this sensing system will be used with the commercial telesurgical robot in our lab (ZEUS Surgical Robot System, Computer Motion, Inc., Goleta, Calif.), we may rely on the surgeon's anatomical knowledge to determine a good initial starting point. For the IMA harvest, the obvious place to begin is the proximal end of the IMA, where it is often visible as it emerges from the pleura. Alternatively, the surgeon may use visuallyguided dissection to find an initial point.

Many methods may be used to detect the pressure variations that designate the artery location. The strategy we selected simply evaluates the maximum and minimum pressure, as our preliminary studies indicate that the arterial pressure is the largest signal variation during the acquisition interval if the sensor is not moved (Figure 2). Other approaches, such as frequency-based techniques, require longer sampling intervals, although they may provide greater accuracy in dynamic or noisy environments. The detection process may also be simplified through the use of an electrocardiogram signal,
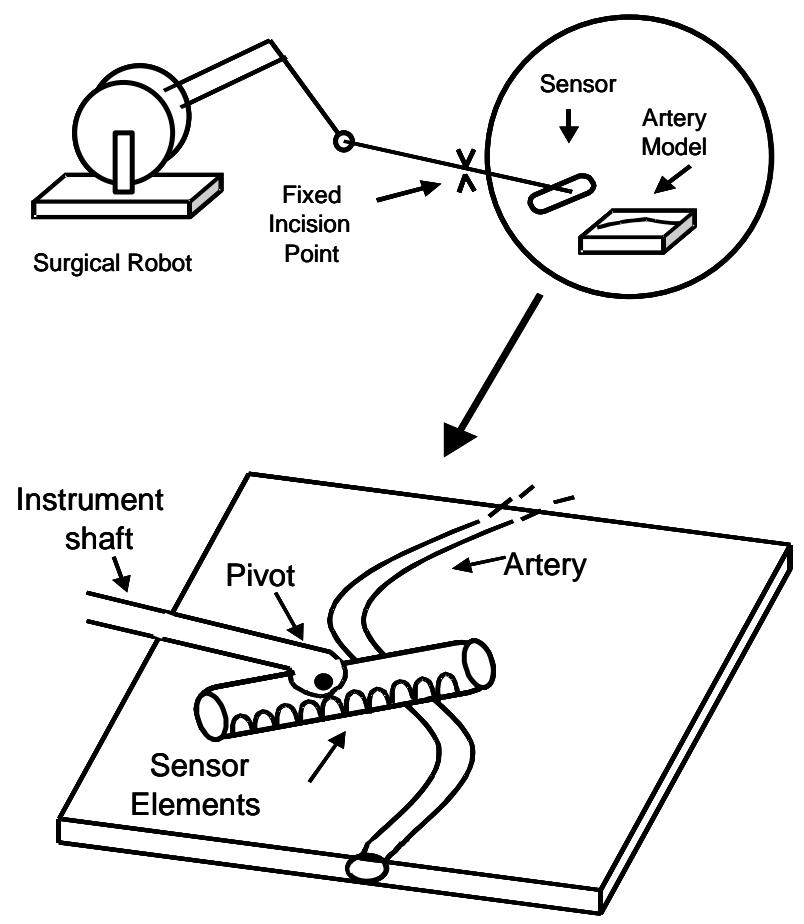

Figure 1. Experimental setup.

which would indicate the timing of the pressure maximum and minimum. We have found that this information may be extracted from the sensor signal itself, however, which avoids the need to connect ECG and robot systems.

In our implementation, the initial detection of the arterial signal begins with four seconds of sampling at $100 \mathrm{~Hz}$. Filtering the data using a $4^{\text {th }}$ order Hamming filter with corner frequencies of 0.5 and $2.0 \mathrm{~Hz}$ provides noise reduction.

The element closest to the artery, $i^{*}$, satisfies

$$
\max \left(a_{i^{*}}\right)-\min \left(a_{i^{*}}\right)=\max _{i}\left(\max \left(a_{i}\right)-\min \left(a_{i}\right)\right)
$$

for $i=1,2, \ldots 10$, where $a_{i}$ is a vector of the readings from the $i$ th sensor element. Any grouping of points over the threshold that abut the beginning or end of the four second sample are ignored because they may represent only a partial sample of the pulse cycle. A threshold is applied to the remaining data from that element,

$$
b_{j}=\left\{\begin{array}{cc}
1, & a_{i^{*} j}>(2 / 3)\left(\max \left(a_{i^{*}}\right)-\min \left(a_{i^{*}}\right)\right)+\min \left(a_{i^{*}}\right) \\
0, & \text { otherwise }
\end{array}\right\}
$$

where $a_{i^{*} j}$ is the $j$ th data point in $a_{i^{*}}$, and $b_{j}$ is the result of thresholding one data point. Empirical tests showed that a threshold value two-thirds of the difference between the minimum to the maximum value provided good results.

The start and stop times are calculated for each of the $\mathrm{n}$ contiguous set of points over the threshold, and averaged to yield a center point in time, $c_{k}$, for each of the 
$k$ pulses. The pulse period, $P$, is estimated by averaging the time between center points,

$$
P=\frac{1}{n} \sum_{k=1}^{n}\left(c_{k+1}-c_{k}\right) .
$$

Because anticipated pulse rates are in the range 1.0$1.5 \mathrm{~Hz}$, the four second sample provides at least two complete peaks. Each time a new artery position is determined, three seconds of data are gathered and processed similarly. The results therein are averaged with the current estimate of pulse timing to continually update the estimate.

Next, the second phase of signal processing determines the location of the artery on the sensor. The IMA is less than $3 \mathrm{~mm}$ in diameter, and to avoid injury it is usually dissected free within a $1 \mathrm{~cm}$ diameter pedicle of adjacent tissue. Localizing the artery to within one or two sensor elements thus proves sufficient; a sub-element interpolation technique would be straightforward to implement if greater accuracy is required for other surgical procedures. The algorithm begins by acquiring a set of 15 sensor samples for each element at the pulse peak time and 15 samples halfway between peaks, i.e. near the pressure minimum. The comparison used to determine whether a sensor was over the artery is a simple difference comparison,

$$
\left(\hat{a}_{i^{*}}\right)_{\text {peak }}-\left(\hat{a}_{i^{*}}\right)_{\text {offpeak }}>\stackrel{?}{>} 2 \sigma
$$

where $\left(\hat{a}_{i^{*}}\right)_{\text {peak }}$ is the 15 point average of the maximally-changing sensor during a pulse peak time, $\left(\hat{a}_{i^{*}}\right)_{\text {offpeak }}$ is the 15 point average of the same sensor halfway between pulse peak times, and $\sigma$ is the standard deviation of the noise.

To indicate the expected direction of the artery path, the surgeon then moves the sensor to a second point on the artery and another set of 15 samples at pulse peak and 15 samples at pulse minimum are acquired. The signal processing algorithm then calculates the location of the artery as above. Following determination of at least two artery locations, the control algorithm extrapolates the artery path and determines the next desired position of the sensor. The extrapolation technique used here is linear regression (involving up to the last four locations); trials with higher-order regression methods showed poor performance due to noise and low position resolution of the artery localization algorithm.

The extrapolation scheme uses a simple adaptive step size to optimize the artery tracking process. Once the sensor moves within $1 \mathrm{~mm}$ of the desired position, the system acquires data for the artery localization algorithm up to seven times in order to locate the pulse. If it succeeds, the new desired position is increased by a small factor over the previous extrapolation; our tests use a modest five percent increment for each successful step. If the system does not locate the pulse, the distance to be moved is halved and applied again to the last location where the system detected the pulse. In this manner, the program increases speed when appropriate and backtracks if it cannot find a pulse.

\section{Experimental Methods}

To simplify testing of the artery tracking system, these tests used a laboratory testbed in place of our surgical robot system. The manipulator is a Phantom robot (Model 1.5, SensAble Technologies, Inc., Woburn, Mass.), with three actuated degrees of freedom. To emulate a minimally invasive surgery configuration, the last joint of the Phantom wrist gimbal was removed and the remaining gimbal joints were attached to a $49.5 \mathrm{~cm}$ long, $4.5 \mathrm{~mm}$ diameter rod that served as the surgical instrument shaft (Figure 1). The rod passes through a fixed pivot, simulating the incision into the patient's thorax. The shaft was attached to the sensor body with a simple pin joint; removing the last wrist gimbal axis precluded rotation of the instrument about the shaft, in order to maintain the correct orientation of the sensor elements.

A personal computer controlled the robot and hosted the data acquisition board. The robot position was controlled using a simple PD controller. To augment the Phantom's fairly small continuous force capabilities, a weight of $0.3 \mathrm{~kg}$ was attached to the instrument shaft just above the sensor.

To investigate the system's performance in a controlled setting, the first tests used a model of the artery and surrounding tissue. The "artery" was a latex tube with a $5 \mathrm{~mm}$ outer diameter and $1 \mathrm{~mm}$ tube-wall thickness attached to a solenoid valve. The tube was inflated to $100 \mathrm{kPa}$ then deflated to the atmosphere at approximately 1 second intervals. This tube was embedded in a flat sheet of foam rubber to simulate the tissue surrounding an artery.

We ran trials on a variety of artery paths, starting with an S-shaped curve $26 \mathrm{~cm}$ in length. Subsequent tests involved seven trials on each of three different artery paths. The paths consisted of a $26 \mathrm{~cm}$ length of tubing on a circular arc with $80 \mathrm{~mm}, 145 \mathrm{~mm}$, and $220 \mathrm{~mm}$ radius. The initial two manual sensor placements were approximately the same for each trial within a single arc radius, and were separated by 1.0 to $2.0 \mathrm{~cm}$ along one end of the artery.

We also conducted preliminary tests on transcutaneously tracking the location of the radial artery in the human wrist. The arm was immobilized to reduce inadvertent motion. The initial sensor placements were approximately $5 \mathrm{~mm}$ apart on the distal end of the artery above the radial styloid process. To estimate the actual location of the artery, the experimenter manually detected the location of the artery with fingertip palpation. The 


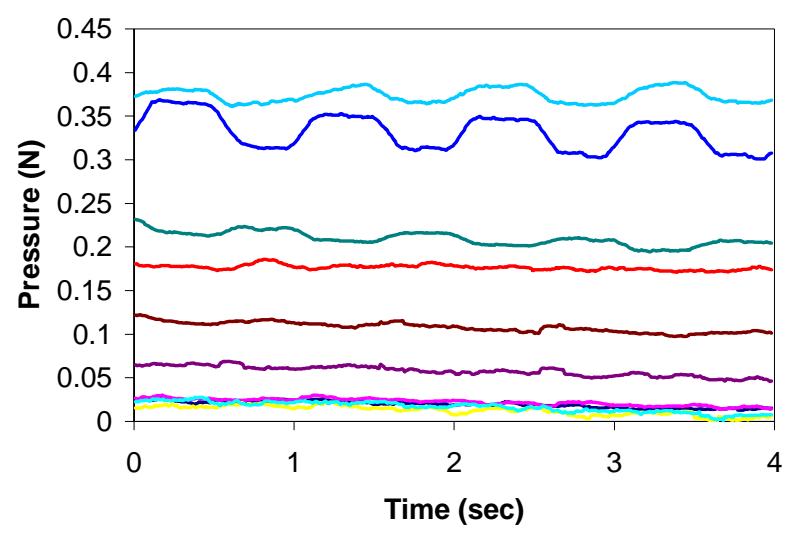

Figure 2. Filtered sensor data.

location of the fingertip during this process was tracked by contact with the end of the Phantom.

\section{Results}

Figure 2 shows a typical four second sample of filtered sensor data when the sensor is centered on the artery model. Two of the elements can be seen to vary up to $0.06 \mathrm{~N}$ every second. A third element varies out of phase with the first two, due to the sensor lifting slightly off surrounding material by the artery pressure. We also observe this effect when sensing the human radial artery under some conditions.

Figures $3 \mathrm{a}$ and $\mathrm{b}$ demonstrate the results of two typical trials with the model artery. The actual artery position is marked, along with the locations determined
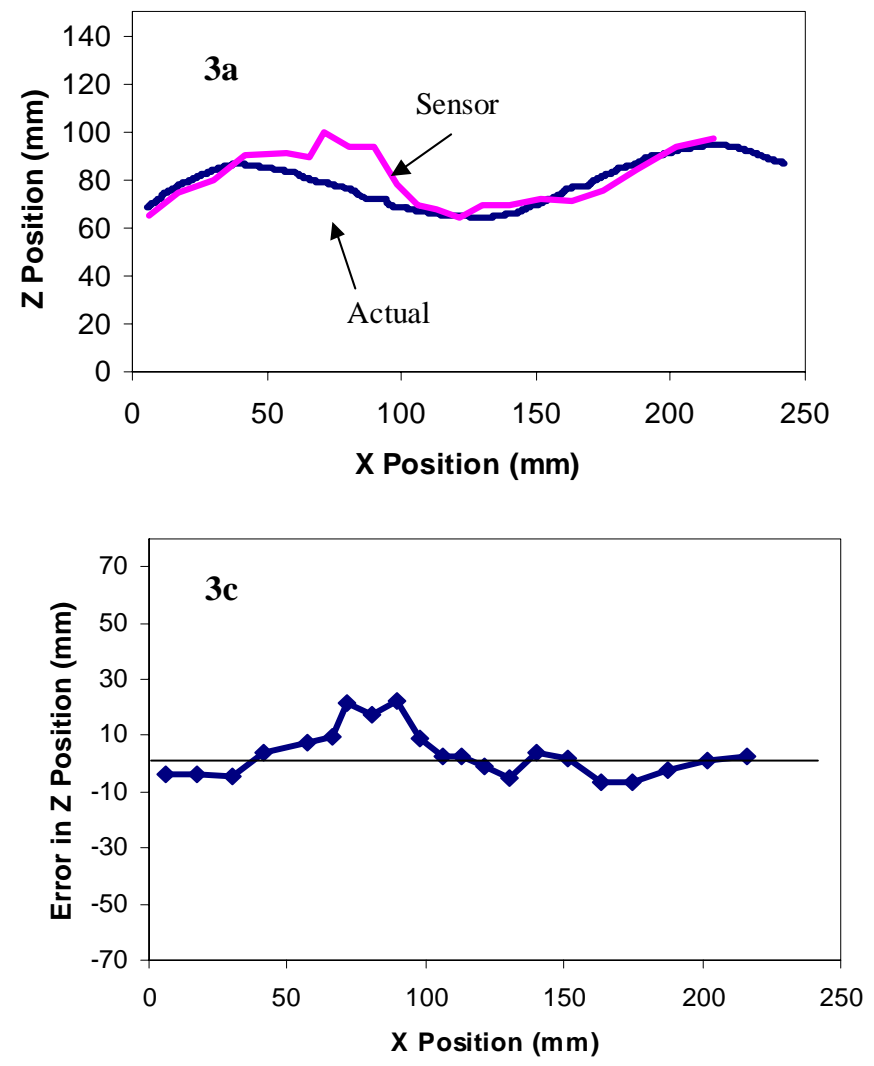

by the program to be on the artery. Figures $3 \mathrm{c}$ and $\mathrm{d}$ graph the error in the $z$-direction (towards the top of the figure) at each position chosen by the program for those two trials. The mean error for both trials was $1.3 \mathrm{~mm}$, with a standard deviation of $7.6 \mathrm{~mm}$ for 33 points.

Figure 4 demonstrates the program's adaptive step size. The program used the first three points to extrapolate to the fourth point, about $3 \mathrm{~cm}$ away from the third point. At the fourth position, no pulse was detectable. The program then backtracked about $1.5 \mathrm{~cm}$ and successfully located the artery. This plot also illustrates the error in the robot's position controller, due to unmodeled friction and compliance.

Figure 5a shows the three artery curvatures. Figures $5 \mathrm{~b}, \mathrm{c}$, and d show the raw data for each trial. The standard deviations of error were $1.93,3.10$, and $3.95 \mathrm{~mm}$ for the $220 \mathrm{~mm}, 145 \mathrm{~mm}$, and $80 \mathrm{~mm}$ radius arcs, respectively. Backtracking occurred approximately once per $80 \mathrm{~mm}$ trial, and during one of the seven $145 \mathrm{~mm}$ trials, but not during any of the $220 \mathrm{~mm}$ trials. All of the trials completed successfully.

Figure 6 displays typical preliminary results for tracking the human radial artery. The points determined by the program to lie on the artery are compared with the position of the artery as localized manually. The zero position in the $x$ direction represents the most distal point that a pulse could be found. The positive $x$ direction is proximal, and the positive $z$ direction is medial.
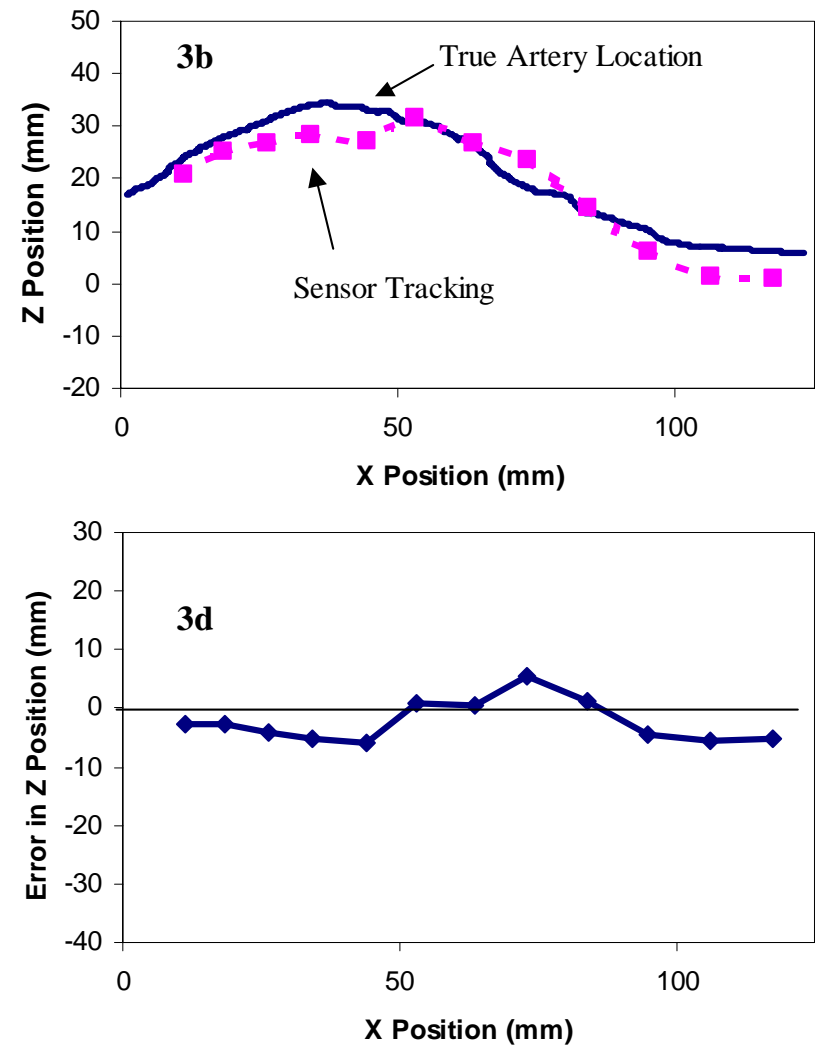

Figure 3. Typical trials and corresponding errors. 


\section{Discussion}

These experimental results provide an initial validation of the semi-autonomous artery tracking system. Although based on simple signal processing, the system succeeded in discriminating the arterial pressure signature from the pressure generated by adjacent tissue. Tracking was successful in the model for radii of curvature as small as $80 \mathrm{~mm}$. The results show a mean error of less than $2 \mathrm{~mm}$ and a standard deviation of about $3 \mathrm{~mm}$. The human radial artery was accurately localized for about $3 \mathrm{~cm}$, demonstrating that the approach is applicable to in vivo conditions as well.

One problem evident in the experimental results is that manipulator compliance occasionally caused the sensor to lose the artery path, despite the backtracking algorithm. When substantial elastic deflections of the instrument and "incision" point occur, the kinematic mapping between the robot wrist and the sensor becomes increasingly inaccurate. In addition, the position controller in the Phantom robot used low gains to enable smooth sliding over the tissue surface. This also contributed to errors in sensor positioning.

Several solutions to these problems are apparent. For example, the instrument shaft can be stiffened to minimize deflections. In addition, the controller can compensate for the observed compliance, through integral gain terms in the control law or predictive models of the
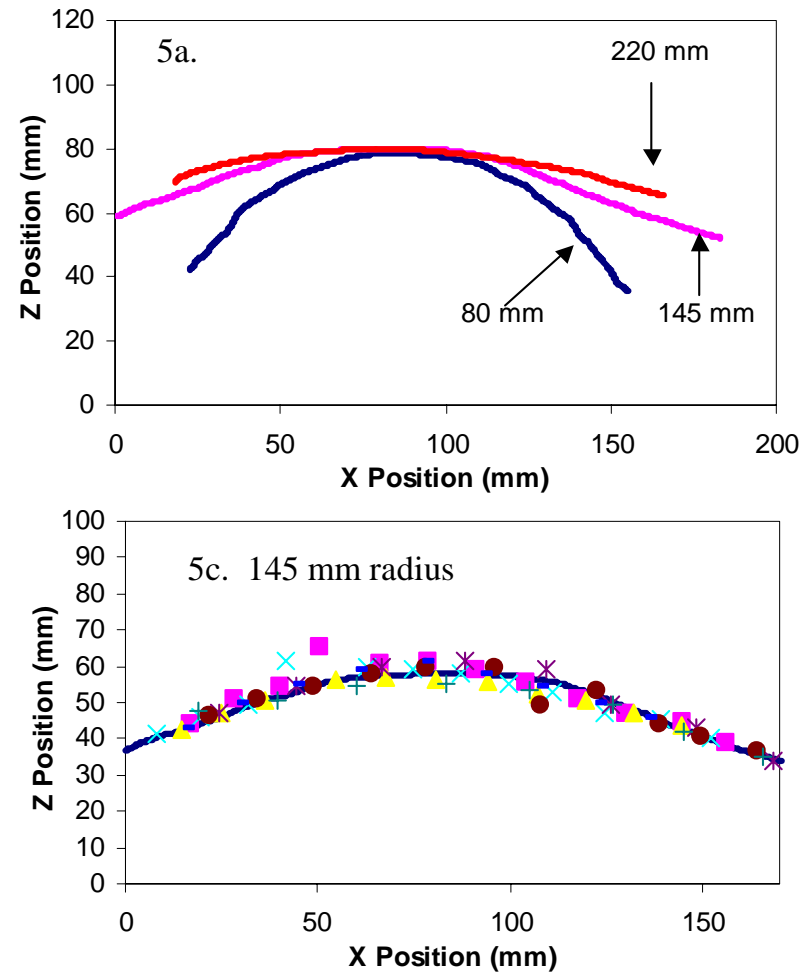

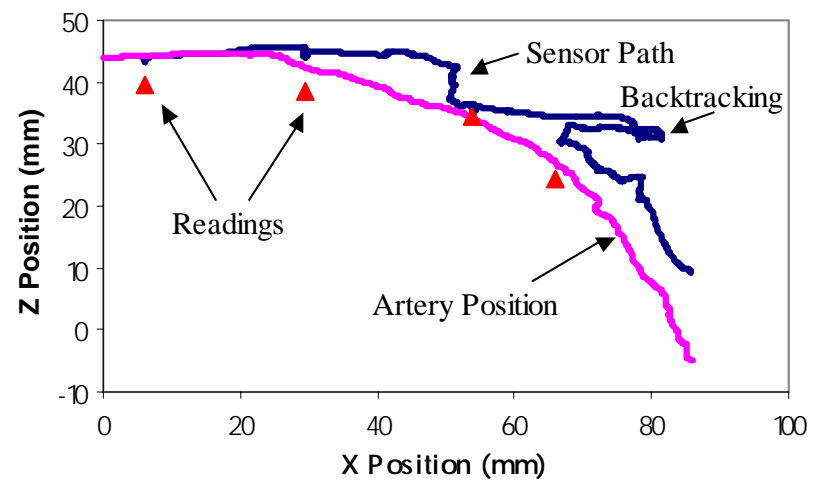

Figure 4. Backtracking example.

system deflection, based on mechanical modeling or empirical observations. We are now working to implement these enhancements.

This compliance problem must also be addressed at a more fundamental level, however. The kinematic model does not now use the angular sensors in Phantom wrist gimbal, but simply assumes a fixed incision point. The additional angular information would allow the kinematic model to compensate for deflection of the incision point. As motion of the incision point is commonly observed in robotic IMA harvest (particularly at the ends of the IMA where the instrument makes a shallow angle with respect to the chest wall), the use of wrist sensors would address a problem that exists with current surgical robotic systems.

Similarly, the issue of controller gain, or more
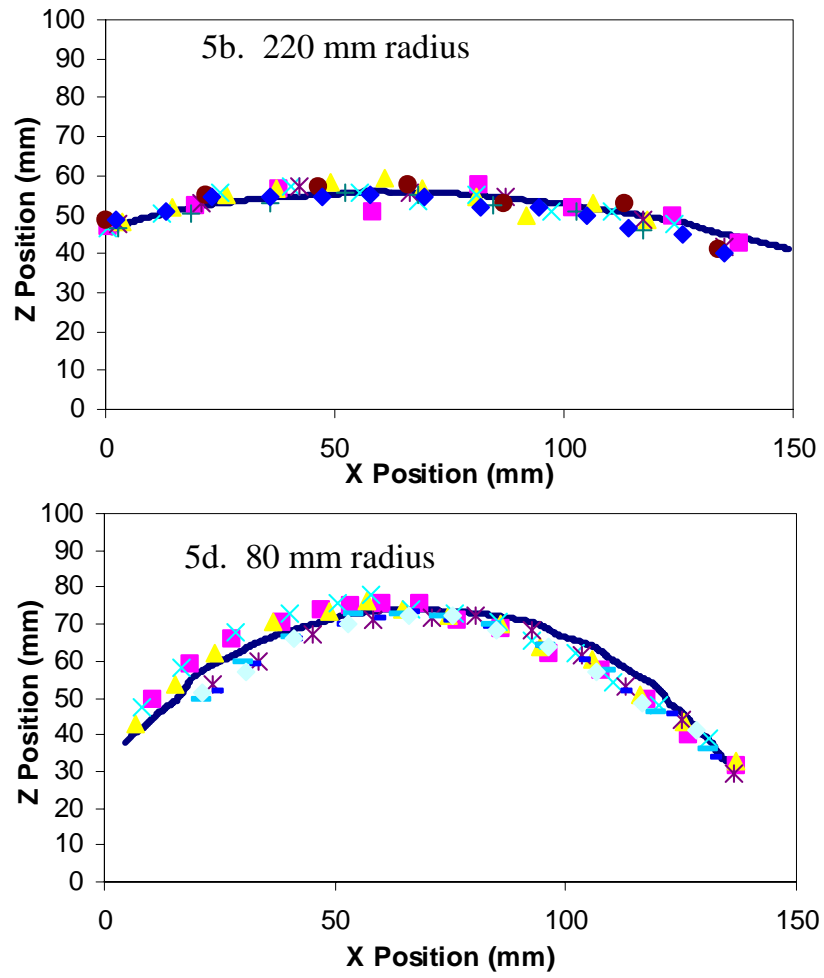

Figure 5. Circular arc artery model sensor position data. Each of the seven trials is indicated by a different symbol. 


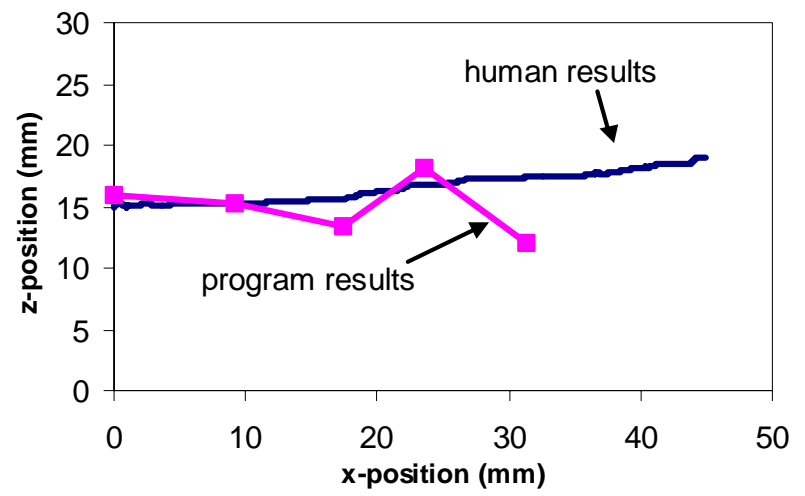

Figure 6. Human radial artery tracking.

generally robot stiffness, is complex. Compliance is essential for this type of sliding task, particularly as it is important to maintain the correct pressure range: too low will not provide an adequate sensor signal, but too high may cause injury, and also fail to generate appropriate signals. For example, replacing the $300 \mathrm{~g}$ mass on the instrument shaft with a $200 \mathrm{~g}$ mass results in normal forces too low to distinguish artery pressure variations from sensor noise. The commercial surgical robots now available for $\mathrm{CABG}$ procedures are fairly stiff, so control methods based on force sensing might be used to generate appropriate compliance. We prefer passive compliant devices built into the sensor instrument, as a safer and easier method. This will entail providing compliance in the normal direction to control contact force, while maintaining stiffness in the lateral directions to allow accurate tracking.

A variety of improvements to the signal processing and tracking algorithms are apparent, although the simplicity of the present implementation has clear advantages in surgical applications. For example, the system now stops moving whenever it samples the tactile sensor. The ability to sample while sliding would accelerate the tracking process, but would require substantially more elaborate signal processing to overcome the pressure fluctuation "noise" inherent in the sliding process. As telerobot-assisted harvest of the IMA now takes 45-60 minutes and the time to track the artery in our model system is typically on the order of two minutes, the value of increased tracking speed is minimal.

Further work must be addressed, however, to understanding the detailed conditions in the surgical setting. For example, the ability to correctly orient the linear array is crucial for successfully tracking the artery. Rotating the instrument shaft could effectively orient the current 1-D sensor array over approximately a 30-degree arc, but more rotation would separate the sensor elements from the surface of the tissue. A 2-D sensor array may thus be helpful or essential, depending on the incision location relative to the tissue surface of interest. Sensor shape and signal-to-noise requirements must also be determined. A variety of packaging issues are apparent, such as the need to sterilize the sensor and to fit through $1 \mathrm{~cm}$ incision ports.

This system represents one example of the use of tactile information in minimally invasive surgery. While surgeons have demonstrated that many procedures are possible without haptic perception, palpation plays an important role in many open surgical procedures, such as using compliance to differentiate healthy or diseased liver tissue, and locating lumps in lung or liver resection (Howe et al. 1995; Peine 1998). Based on our growing understanding of the role of tactile sensation in human manipulation, it is reasonable to conjecture that the sensing instruments that facilitate artery tracking may also enable a broad range of surgical tasks.

\section{References}

Fearing R. S. (1990). Tactile sensing mechanisms. Intl. J. Robotics Research 9(3):3-23.

Fischer H, Trapp R. (1996). Tactile optical sensor for use in minimal invasive surgery. Stud Health Technol Inform 29:623-9.

Harris ML, Morberg P, Bruce WJ, Walsh WR. (1999). An improved method for measuring tibiofemoral contact areas in total knee arthroplasty: a comparison of K-scan sensor and Fuji film. J Biomech 32(9):951-8.

Howe RD, Peine WJ, Kontarinis DA, and Son JS (1995). Remote palpation technology. IEEE Engineering in Medicine and Biology 14(3):318-323.

Matsumoto S, Ooshima R, Kobayashi K, Kawabe N, Shiraishi T, Mizuno Y, Suzuki H, Umemoto S. (1997). A tactile sensor for laparoscopic cholecystectomy. Surg Endosc 11(9):939-41.

Park S. S., Howe R.D., and Torchiana D.F. (2001). "Virtual Fixtures for Robot-Assisted MinimallyInvasive Cardiac Surgery," Medical Image Computing and Computer-Assisted Intervention MICCAI 2001, ed. by Niessen W.J., Viergever, M.A. Springer-Verlag, Berlin:1419-1420.

Pawluk D.T.V., Son J.S., Wellman P.S., Peine W.J., and Howe R.D. (1998). "A Distributed Pressure Sensor for Biomechanical Measurements," ASME Journal of Biomechanical Engineering 102(2):302-305.

Peine W.J. (1998). Remote palpation instruments for minimally invasive surgery. Ph.D. Thesis, Division of Engineering and Applied Sciences, Harvard University.

Schellenberg AG, Marshall MB, Salgo IS. (2001). Intraoperative ultrasound for localization of patent left internal mammary artery grafts in repeat cardiothoracic surgery. J Cardiothorac Vasc Anesth 15(2):228-30.

Stephenson ER Jr, Sankholkar S, Ducko CT, Damiano RJ Jr. (1998). Robotically assisted microsurgery for endoscopic coronary artery bypass grafting. Annals Thoracic Surg. 66(3):1064-67. 